\title{
Coastal monitoring with high resolution synthetic aperture radar
}

\author{
P. Trivero, W. Biamino, M. Borasi \& M. Cavagnero \\ DISAV - Università del Piemonte Orientale, Italy
}

\begin{abstract}
Coastal areas are dynamic zones affected by natural change and human use: they contain rich resources to produce goods and services and are home to most commercial and industrial activities, as well as fragile and valuable ecosystems.

A continuous monitoring activity is essential to prevent environmental threats that can be very dangerous for such a sensitive ecosystem. Satellite-borne sensors are valuable tools for this purpose, particularly the Synthetic Aperture Radar (SAR), which is able to operate by day and night in any weather conditions. In the past, the limited revisit time was the main drawback of SARs, because of the small number of operating sensors. In the past year several new satellites have been launched, with acquisition modes characterised by large swaths; now a daily revisit is possible for high and mid-latitude areas included between the tropic and the Arctic Circle. Moreover, spatial resolutions available from new sensors are so high (up to 1 meter per pixel) that it is now possible to observe details in close proximity of coastal zones.

We present a monitoring system under development, based on multi-sensor SAR from which we extract marine features such as high resolution wind and wave fields and sea surface pollution. Methodologies and operational exempla are described.

The system is primarily designed for monitoring activities at regional and local scales. Focusing on a particular area, it is possible to gather and integrate all available data for a comprehensive observation and management of both routine and emergency events.
\end{abstract}

Keywords: sea surface slicks, coastal wind, SAR remote sensing. 


\section{Introduction}

Countries with extended coastlines are often threatened by sea pollution: a continuous monitoring activity is therefore important to set up prevention actions and to detect early any risky events.

High resolution SAR images offer a good opportunity for this purpose. The SAR is a powerful instrument to detect the presence of surfactants and is able to operate regardless of sunlight and weather conditions. The SAR sends microwave pulses and collects the echoes from sea; by processing the backscattered signals, a high spatial resolution image is produced. Radio pulses are sent with incidence angles between 20 and 60 degrees and are backscattered by sea surface roughness. Sea surface roughness is produced by wind and it is composed of short waves $(\sim 1-10 \mathrm{~cm}$ wavelength). The detected signal is due to constructive interference coming from these short wavelengths. The basic mechanism involved is the normalised radar cross-section (NRCS) which, for incidence angles higher than $20^{\circ}$, is proportional to the spectral energy density of the sea waves having wavelength $\Lambda$ that obey the Bragg resonance condition (1):

$$
\Lambda=\frac{\lambda}{2 \sin \theta}
$$

where $\lambda$ is the radar wavelength and $\theta$ the incidence angle of radar beam. For low incidence angles the backscatter is due to specular reflection.

The presence of a surface film causes a damping of these waves; an area covered by slick appears "flatter" than the surrounding sea. The sea waves, which are Bragg resonant with microwaves employed by the SAR systems, fall in the short gravity wave region where the damping, due to a presence of a slick, is maximum $[1,2]$. However, dark areas can be also due to atmospheric effects such as low wind [3, 4].

A methodology to detect sea surface slicks is described, able to discriminate among low wind, oil spills, biogenic and anthropogenic slicks.

\section{Data availability}

Over the last decades, satellite-borne SARs are used for earth observation. However, up to the turn of the century in 2000 the limited number of satellites made it impossible to have an elevated frequency of passages over the same area.

Today, instead, many satellites or even constellations are available. Among them all, the European ERS-2 and Envisat offer spatial resolution up to 30 meters per pixel at various polarisations and acquisition modes; similar performances are reached by the Japanese ALOS. Higher resolutions are available from new sensors such as the Canadian Radarsat-2 $(3 \mathrm{~m} / \mathrm{pixel})$ or the German Terrasar-X $(1 \mathrm{~m})$. The Italian Space Agency manages a constellation named COSMO Sky-Med of three satellites with spatial resolution up to $1 \mathrm{~m}$, and the fourth satellite is planned for launch in 2010 [5]. 
The revisit time, for every satellite, depends on devices characteristics, acquisition geometry, as well as on geographic coordinates of the observed point (at higher latitudes acquisitions are more frequent). Using the method proposed by Smith [6], a mean revisit time of about $4-5$ days is expected when using a single satellite at mid - high latitudes. Combining two satellites this value can drop to a couple of days; with today's satellite availability is expected to be a daily revisit on most regions, allowing the use of SAR as a tool for routinely observations.

\section{Wind and pollutant extraction technique}

The proposed methodology under development is based on rationale on which we have developed in the last few years an oil spill detection algorithm. This algorithm is described in detail in [7, 8]. The new procedure extends the capabilities of the previous method: a preliminary classification is carried out based on wind field evaluated in two independent ways; further it discriminate not only oil spills but also the look-alikes into two different sub-categories: biogenic and anthropogenic slicks.

The procedure expects the systematic acquisition of SAR data from available satellite imagery of the coastal area to be monitored. There are two operating modes: the first consists in a statistical study of winds and pollution in the area by means of historical data and the other one includes the monitoring of the area in quasi real time.

The steps of the detection procedure are summarised in the following.

\subsection{Wind field evaluation}

A high resolution meteorological model is used to evaluate the direction and the intensity of the wind. We utilised the Weather Research and Forecast (WRF) model, version 3.1 [9]. WRF is developed by the National Center for Atmospheric Research (NCAR), the National Oceanic and Atmospheric Administration (the National Centers for Environmental Prediction (NCEP) and the Forecast Systems Laboratory (FSL), the Air Force Weather Agency (AFWA), the Naval Research Laboratory, the University of Oklahoma, and the Federal Aviation Administration (FAA).

In parallel, SAR images are, as a first step, calibrated [10] to retrieve the radar backscattering. The second step consists in the compensation of the attenuation due to the incidence angle [11], because the sea scattering is dominated by the Bragg mechanism which produces a stronger signal in the near range than in the far range. Finally the land, vessels and platforms are masked.

From the pre-processed images, the structures containing wind direction information are extracted [12-14]. The wind direction, deduced by means of Continuous Wavelet Transform (CWT2) allows the wind intensity from the SAR NRCS to be obtained by applying the CMOD5 model [15]. The wind vector direction ambiguity is solved by the process of dealiasing, described in [14] or by using wind direction from the meteorological model. 
At present the wind field is obtainable from the SAR images in $\mathrm{C}$ band, but similar models are in preparations for $\mathrm{X}$ and $\mathrm{L}$ band. The application of CMOD5 can improve the meteorological model datum and the information about the high resolution wind field near the coast is provided. The wind field allows the evaluation of the intensity of the coastal waves, the induced surface current and therefore the transport of the aerosols on the coast.

\subsection{Detection procedure}

The comparison between the wind intensities, obtained by the meteorological model and by analysis of the SAR image, should generally show comparable values; such values can be however very different in presence of a dark area in the SAR images when the roughness is damped by the presence of a surfaceactive film.

CMOD computes wind speed from the NRCS, assuming a given relationship between wind and sea surface roughness; therefore, where damping is due to an oil spill, or to a slick of biogenic or anthropogenic origin, the result has no physical meaning. At medium and high resolution, a similar problem is due to ships present in the scene, noticeable by the high reflection.

As an example, figure 1(a) shows an oil spill. Wind speed, evaluated from SAR image, is $4.1 \mathrm{~m} / \mathrm{s}$ outside the slick and $0.4 \mathrm{~m} / \mathrm{s}$ inside. The WRF model predicts a $4.5 \mathrm{~m} / \mathrm{s}$ wind on the whole area. In the case shown on figure 1(b), ship is visible throughout the image as bright spot; the computed wind is about 20 $\mathrm{m} / \mathrm{s}$, another value with no physical significance. For this reason a "ship removal" procedure has been added by discarding pixels having reflectance higher than those contiguous.

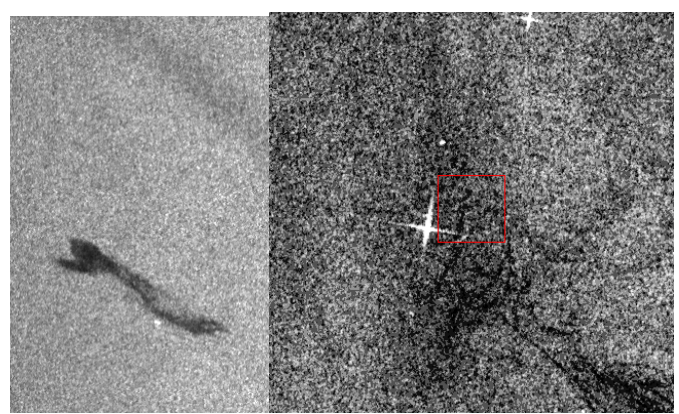

(a)

(b)

Figure 1: $\quad$ Examples of oil spill and ships in SAR images.

The first steps of the procedure are the land masking, the ship masking and the identification of dark areas as "candidate slicks"; then the wind intensity value is evaluated in the barycentre of every dark area (by averaging values of the nearest cells) using both systems: meteorological model and SAR analysis. These values are compared and if the meteorological model provides a value higher than $20 \%$ with respect to the intensity obtained by SAR we establish that 
dark area is due to a film of a substance that damps the surface roughness; otherwise, if the values are both low and comparable the area is classified as "low wind area".

On the remaining candidates, the oil spill detection algorithm is applied allowing the discrimination between "oil slicks" and "look-alikes". This detection system is based up on a probabilistic method that distinguishes oil spills from other similar sea surface features in SAR images by evaluating the radiometric and the geometric characteristics of the areas under test. In order to minimize the operator intervention, it adopts automatic selection criteria to extract the potentially polluted areas from the images. The method, based on a simple classifying algorithm applied to SAR image processing $[7,16]$, is able to discriminate oil spills among all dark areas containing look-alike surface phenomena and has an a priori percentage of correct classification higher than $90 \%$ on a different dataset of verified slicks. Figure 2 shows examples of oil spills (left) and look-alikes (right).

Before the operational use, the algorithm has been tuned with a training dataset, composed by about 400 selected images containing both oil spills and look-alikes, confirmed by an expert photo interpreter.

On the dark areas several measurements are performed and results are compared values with typical values, computed on oil spills and look-alikes from the training dataset, in order to compute the probability, for every candidate, to be an oil slick.

The lookalikes are then analysed in a similar way. A training dataset has been built, containing both "biogenic" and "anthropogenic" as classified by an expert photo-interpreter who is able to classify dark areas using the following criterion: biogenic slicks have dark areas with smooth turnings often in a shape of a spiral while anthropogenic slicks have higher viscosity and tend to change their shape. For every look-alike a number of parameters are computed:

- $\quad$ surface and perimeter of dark area

- average backscattering and standard deviations inside the area

- average backscattering and standard deviations outside the area

- gradient of backscattering across the dark area

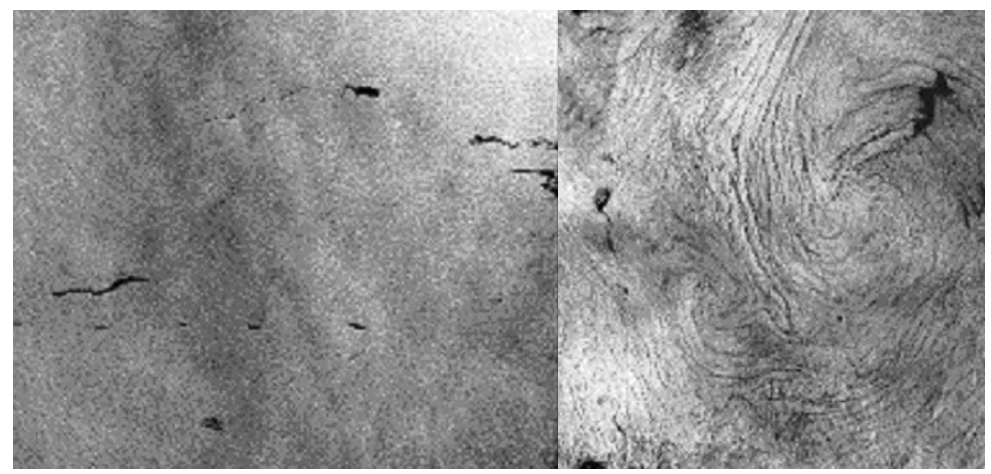

Figure 2: Examples of dark areas in SAR images. 
- form factor

- $\quad$ ratio between perimeter and area

- $\quad$ ratio between average backscattering inside and outside the area

- $\quad$ ratio between standard deviation inside and outside the dark area

- $\quad$ ratio between average backscattering and standard deviation inside the dark area

- $\quad$ ratio between average backscattering and standard deviation outside the area

- $\quad$ ratio between the two last operations

The same approach to discriminate oil spills from look-alikes described in [16] is here applied to separate the look-alikes into the two sub-categories "biogenic" and "anthropogenic" using the compound probability.

\section{Case study}

As an example, we show results from the application of the developed methodology on an Envisat Wide Swath image acquired on June 2008 (figure 3(a)); this image contains almost all detectable features.

By elaborating the image itself (figure 3(a)), wind direction has been extracted applying the CWT method and wind speed (figure 3(b)) with CMOD-5 algorithm. Brighter areas indicate higher wind. In order to have an independent estimation of wind field, results from WRF 3.1 meteorological model have been taken into account. Figure 4 summarizes the situation at regional scale, while values are available at $1 \mathrm{~km}$ resolution.

After land masking and ship removal, dark areas have been identified and wind fields comparison carried out. Figure 5 shows an example of a low wind area as detected by SAR. Here, as expected, the average wind speed evaluated from SAR image is $0.7 \mathrm{~m} / \mathrm{s}$. The WRF model predicts a comparable value $(0.5 \mathrm{~m} / \mathrm{s})$.

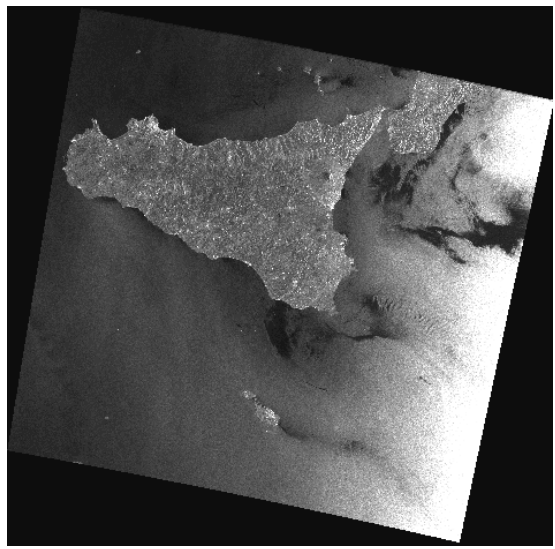

(a)

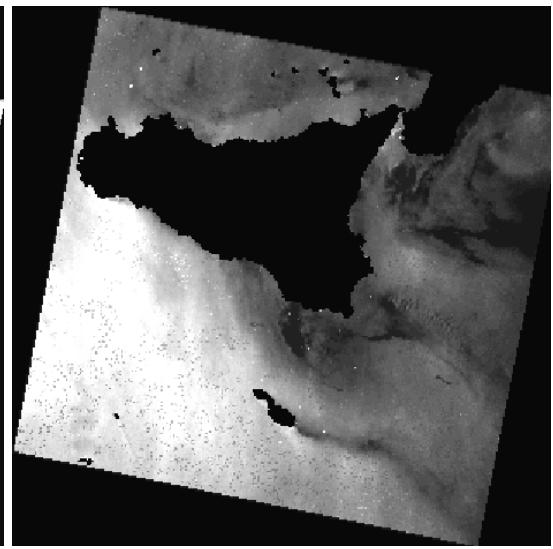

(b)

Figure 3: $\quad$ SAR image (a) and wind intensity (b). 


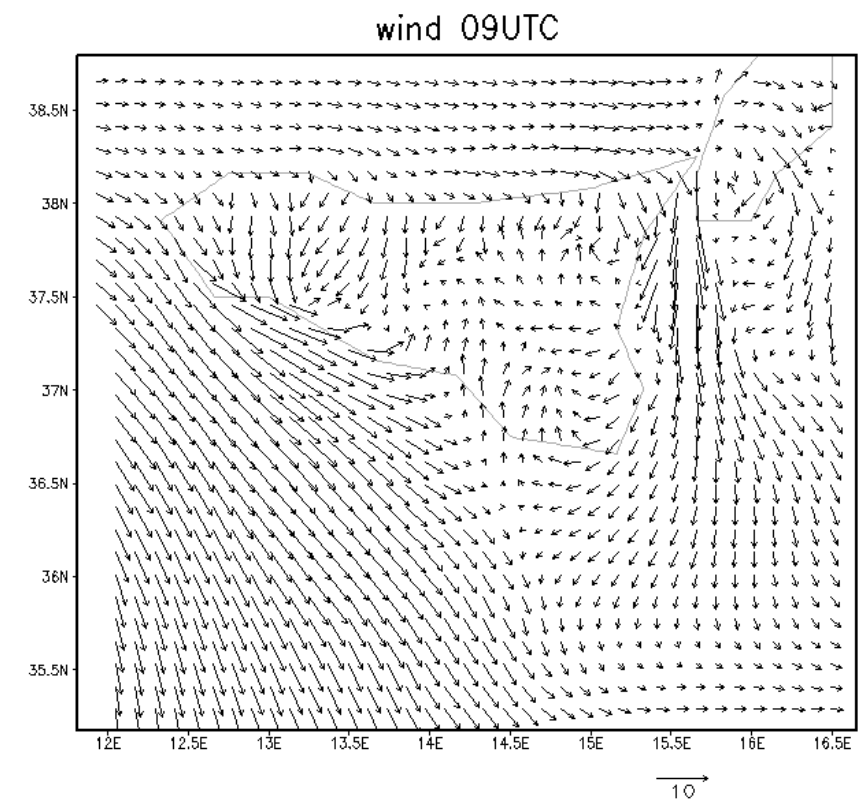

Figure 4: $\quad$ Wind field obtained applying the WRF 3.1 meteorological model.

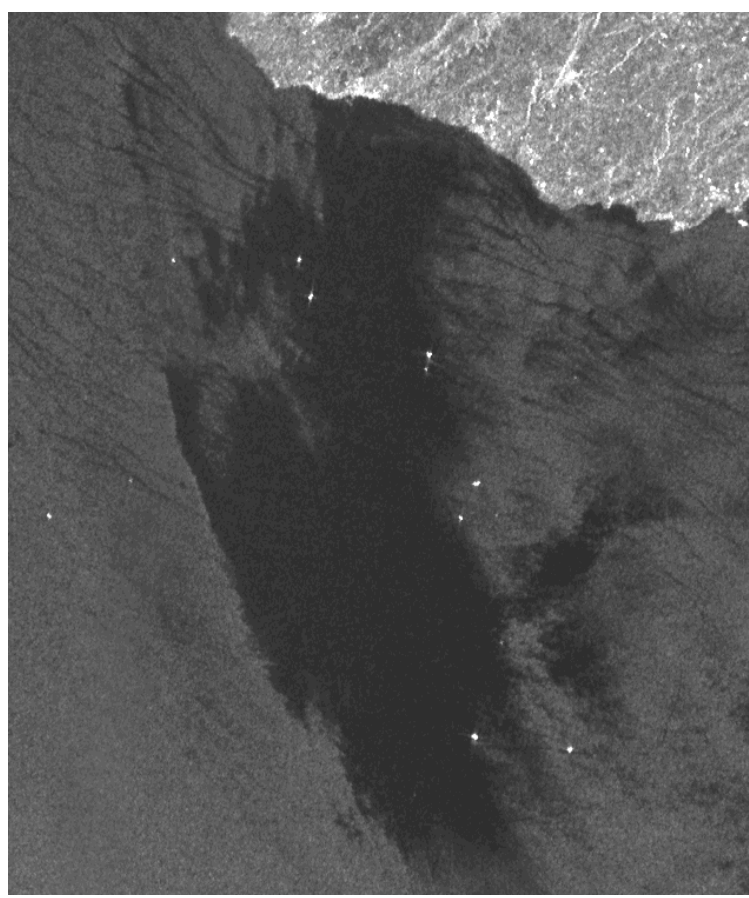

Figure 5: $\quad$ Example of a low wind area as detected by SAR. 


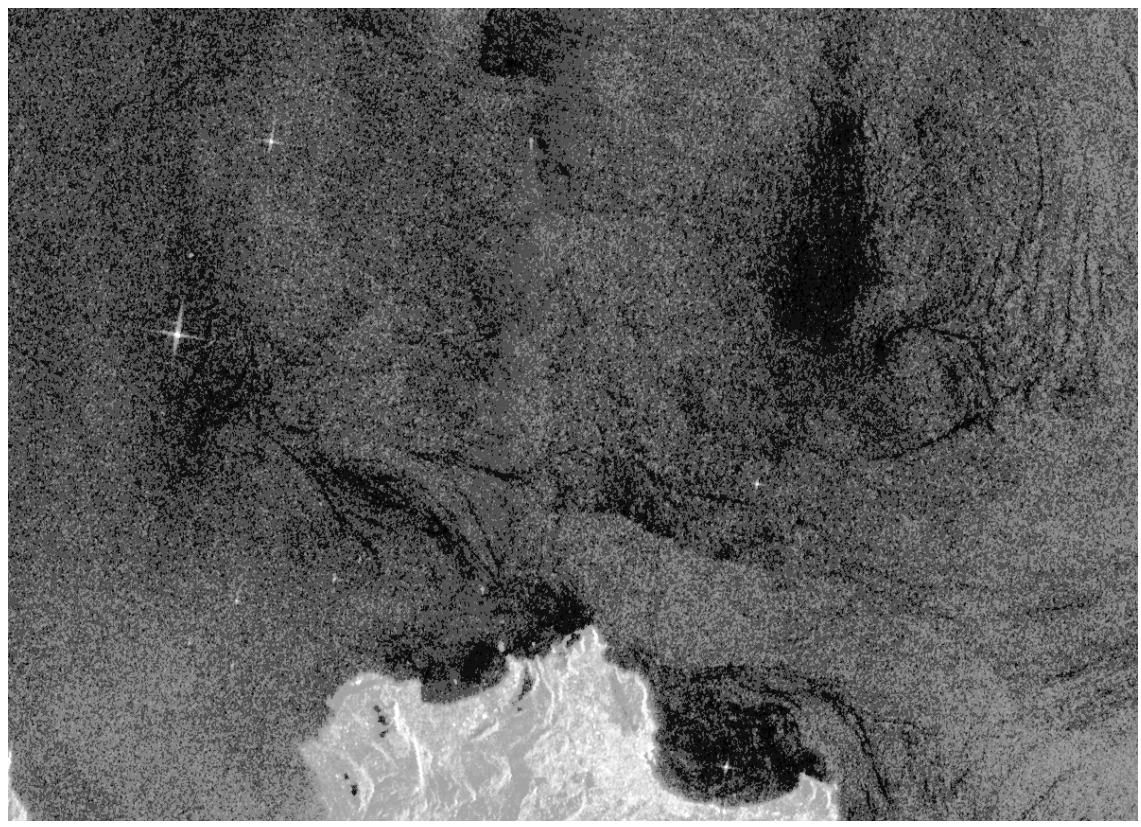

Figure 6: $\quad$ Example of a biogenic slick in a SAR image.

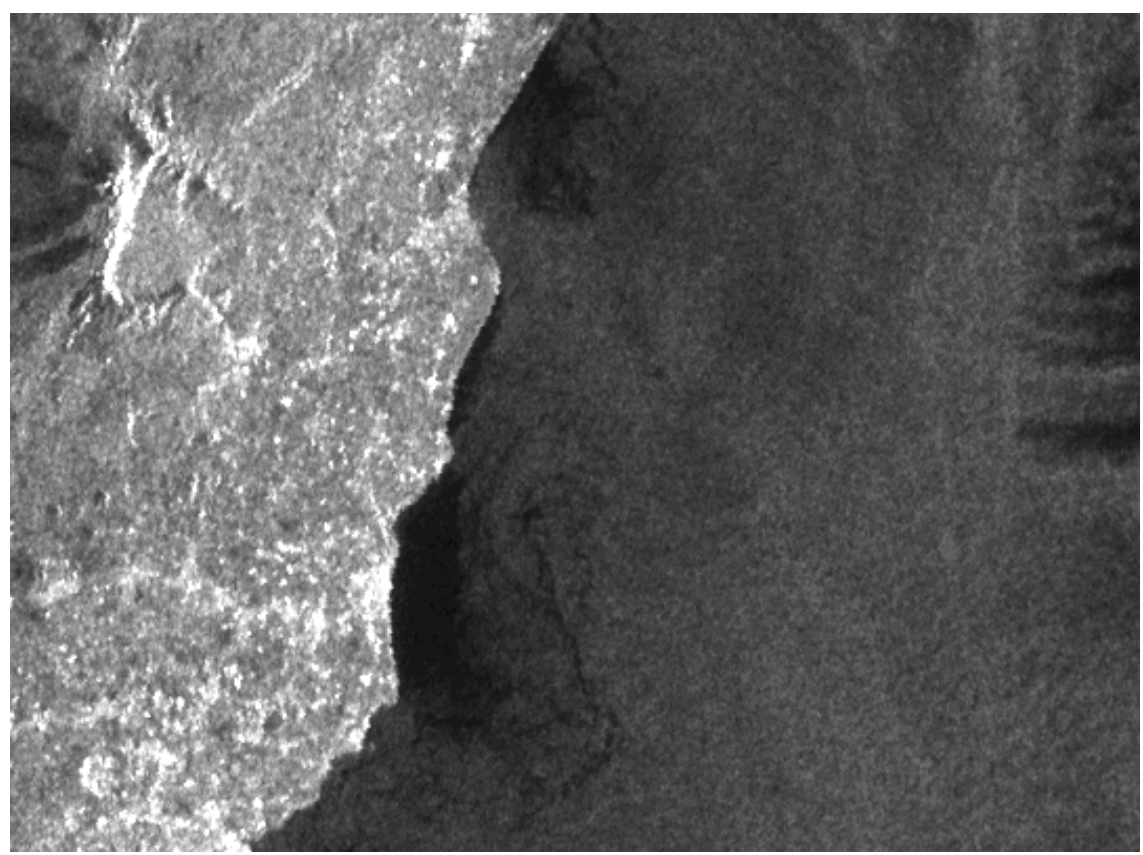

Figure 7: $\quad$ Example of an anthropogenic slick in a SAR image.

WIT Transactions on Ecology and the Environment, Vol 130, (C) 2010 WIT Press

www.witpress.com, ISSN 1743-3541 (on-line) 
On the "slick candidates" the second step is then applied in order to detect the oil spills; an example has been previously shown on figure 1 .

The remaining look-alikes are subsequently analysed allowing the further discrimination. By zooming the SAR image on the observed zone, with an enhanced contrast (figure 6), the structures of a typical biogenic slick can be seen, as classified by the detection algorithm. Average wind speed, evaluated from SAR image on the slicks, is $2.3 \mathrm{~m} / \mathrm{s}$. The WRF model predicts a $2.9 \mathrm{~m} / \mathrm{s}$ wind on the whole area.

An anthropogenic slick shows similar features; in figure 7 the average wind speed, evaluated from SAR image on the slicks, is $1.1 \mathrm{~m} / \mathrm{s}$, while the WRF model predicts a $4.9 \mathrm{~m} / \mathrm{s}$ wind on the whole area.

The analysed cases show how the difference, between the two wind evaluation, can be used as a method to characterise dark areas by discriminating between low wind and slicks; among the slicks, a more refined classification (oil slicks, biogenic slicks, anthropogenic slicks) with the algorithm based on geometric and radiometric characteristics.

\section{Conclusions}

The developed methodology is able to monitor a coastal area supplying wind field and the presence of sea surface pollutants.

A comparison between wind computed from SAR image (with CMOD5) and wind from the WRF meteorological model, can lead to an identification of slicked areas and low wind areas in SAR image.

A previously established technique, used for oil spill detection in SAR images, has been extended to classify all slicks detected in the SAR image applying a probabilistic approach to discriminate between biogenic and anthropogenic (non oil) slicks.

\section{Acknowledgements}

This work has been carried out on the framework of the Announcement of Opportunity for Envisat AO 464 and of the Project Start Up C1P.5404 of the European Space Agency.

\section{References}

[1] Trivero P., Fiscella, B., Gomez, F. \& Pavese, P., SAR detection and characterization of sea surface slicks, International Journal of Remote Sensing, 19, pp. 543-548, 1998.

[2] Brekke, C. \& Solberg, A. H. S., Oil spill detection by satellite remote sensing, Remote Sensing of Environment, 95, pp. 11-13, 2005.

[3] Alpers, W., Measurement of mesoscale oceanic and atmospheric phenomena by ERS-1 SAR, URSI Radio Sci. Bull, 275, pp. 14-22, 1995. 
[4] Melsheimer, C.; Alpers, W \&. Gade, M., Investigation of multifrequency multipolarization radar signatures of rain cells over the ocean using SIRC/X-SAR data, J. Geophys. Res., 103(C9), pp. 18,867-18,884, 1998.

[5] Trivero, P. \& Biamino, W., Observing marine pollution with Synthetic Aperture Radar. Geoscience and remote sensing (in press), InTech, 2009.

[6] Smith, A. J. E., A practical method for computing SAR satellite revisit times: application to RADARSAT-1 and ENVISAT, International Journal of Remote Sensing, 28(6), pp. 1123-1135, 2007.

[7] Fiscella, B., Giancaspro, A., Nirchio, F., Pavese, P. \& Trivero P., Oil spill detection using marine SAR images, International Journal of Remote Sensing, 21(18), pp. 3561-3566, 2000.

[8] Nirchio, F., Sorgente, M., Giancaspro, A., Biamino, W., Parisato, E., Ravera, R. \& Trivero, P., Automatic detection of oil spills from SAR images, International Journal of Remote Sensing, 26(6), pp. 1157-1174, 2005.

[9] WRF - the Weather Research \& Forecasting Model http://www.wrfmodel.org/

[10] Laur, H., Derivation of the Backscattering coefficient $\sigma_{0}$ in ERS-1.SAR PRI Products, ESA doc No: ES-TN-RS-PM-HL09, 1992.

[11] Rosenthal, W., Lehner, S., Hortsmann, J. \& Koch, W., Wind measurements using ERS-1 SAR, Proc. of the $2^{\text {nd }}$ ERS Application Workshop, London, UK, 6-8 December 1996.

[12] Zecchetto S., De Biasio, F., Pierdicca, N. \& Trivero, P., Small scale properties of the radar backscattering from the sea surface at off nadir angles, Proc. IGARSS 2001, Sydney, Australia, 2001.

[13] Zecchetto, S., De Biasio, F. \& Trivero, P., Computation of wind direction from SAR images without external a priori information. Proc. IGARSS 2007, Barcelona, Spain, 2007.

[14] Zecchetto S. \& Trivero P. An automatic procedure to retrieve the windfield from SAR Images, Rivista Italiana di Telerilevamento, 41(3), pp. 123-131, 2009.

[15] Hersbach, H., Stoffelen, A. \& de Haan, S. An Improved C-band scatterometer ocean geophysical model function: CMOD5, J. Geophys. Res., 112, 2007

[16] Nirchio, F., Sorgente, M., Giancaspro, A., Pavese, P., Ravera, R. \& Trivero, P., A method to detect oil spill based on SAR images, Proc. Oil and Hydrocarbon spills, Rhodes, 2002. 